\title{
VATS right upper lobe bronchial sleeve resection
}

\author{
Qianli Ma, Deruo Liu \\ Department of Thoracic Surgery, China Japan Friendship Hospital, Beijing 100029, China \\ Correspondence to: Deruo Liu, MD. No. 2, Yinghua East Road, Chaoyang, Beijing 100029, China. Email: deruoliu@vip.sina.com.
}

\begin{abstract}
Background: The aim of this study is to discuss video-assisted thoracic surgery (VATS) sleeve bronchial lobectomy when handling the locally advanced central lung cancer (involving the trachea and/or main bronchus).

Methods: A $2.5 \mathrm{~cm} \times 1.0 \mathrm{~cm}$ mass was found in the right upper lobe. Bronchoscopy demonstrated the tumor obstructing the right upper lobe bronchus and involved the right main bronchus and bronchus intermedius. Interrupted sutures were chosen for bronchial anastomosis. Bronchial membrane was sutured first, and then circumference end-to-end anastomoses were carried out using 3-0 absorbable sutures.

Results: There were no complications and the patient was discharged 8 days postoperatively.

Conclusions: The third intercostal space of the anterior axillary line was suggested for right upper lobe bronchial sleeve resection. This incision can reduce the distance and angle between the anastomosis to the incision, and facilitate anastomosis. This approach can also prevent operator from fatigue for keeping one posture for a long time. Clearance of the mediastinal lymph nodes before cutting the bronchus was helpful for exposing the right main bronchus, the upper lobe bronchus and bronchus intermedius satisfied. And this option would avoid pulling bronchial anastomosis during mediastinal lymph nodes clearance. Interrupted suture was safe and effective for VATS bronchial anastomosis.
\end{abstract}

Keywords: Sleeve lobectomy; video-assisted thoracic surgery (VATS); lung cancer

Submitted Mar 01, 2016. Accepted for publication Mar 31, 2016.

doi: $10.21037 /$ jtd.2016.04.12

View this article at: http://dx.doi.org/ 10.21037/jtd.2016.04.12

\section{Introduction}

Surgery for patients with non-small cell lung cancer (NSCLC) involving proximal bronchi can be challenging. Pneumonectomy is associated with high rate of complications, especially for patients with compromised pulmonary function $(1,2)$. Sleeve lobectomy can save noninvolved lung parenchyma for improving quality of life postoperatively. In 1952, Allison performed the first successful right upper lobe sleeve lobectomy for a patient with bronchogenic carcinoma (3). With the development of video-assisted thoracoscopy surgery (VATS), locally advanced lung cancer, has been adapted for thoracoscopic lung resection and has obtained worldwide acceptance (4-10). However, central lung cancer was still considered a contraindication of thoracoscopic surgery $(11,12)$. Thoracoscopic bronchial sleeve resection has only been reported in limited studies (13-16). This report describes a minimally invasive technique for VATS right upper lobe bronchial sleeve resection.

\section{Clinical data}

A 66 years old men was admitted for coughing for two months. He had smoked for 30 years, 20 packs a day. Chest CT revealed a $2.5 \mathrm{~cm} \times 4.5 \mathrm{~cm}$ mass in the right upper lobe (Figure 1). Bronchoscopy demonstrated the tumor obstructing the right upper lobe bronchus and involved the right main bronchus and bronchus intermedius. Pathology was squamous cell carcinoma. He received two cycles of neoadjuvant chemotherapy (Gemcitabine $2.0 \mathrm{~g}$ Day 1, $8+$ Cisplain $40 \mathrm{mg}$ day $1-3)$. The lesion's size reduced to $2.5 \mathrm{~cm} \times 1.0 \mathrm{~cm}$ (Figure 2). There were no positive past medical history and co-morbidities. His pulmonary function result was FEV1: $1.91 \mathrm{~L}$ (64.7\% predicted); FVC: $4.36 \mathrm{~L}$. He was selected to be candidate for VATS sleeve resection. 


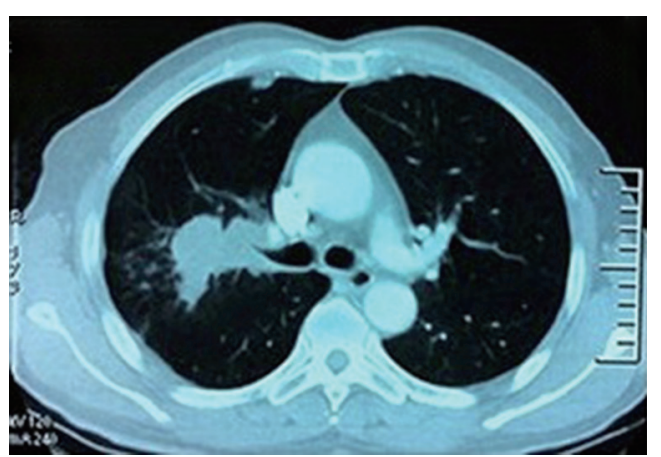

Figure 1 Chest CT revealed a $4.5 \mathrm{~cm} \times 2.5 \mathrm{~cm}$ mass in the right upper lobe.

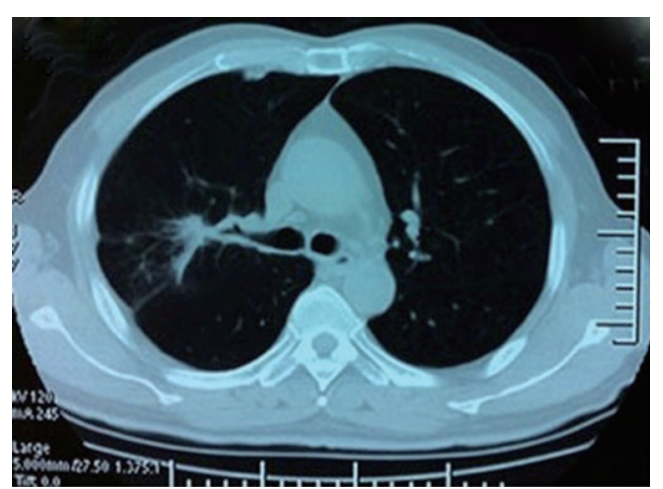

Figure 2 The lesion's size was reduced to $2.5 \mathrm{~cm} \times 1.0 \mathrm{~cm}$ after two cycles of neoadjuvant chemotherapy.

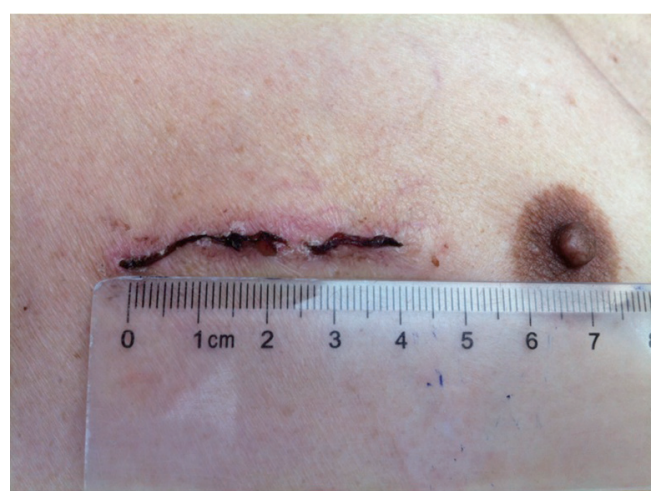

Figure 3 A 4-cm long incision was made in the third intercostal space in the pre-axillary line.

\section{Operative techniques}

Under general anesthesia with double-lumen endotracheal intubation and left lung ventilation, left lateral decubitus position was chosen. His arms extended to $90^{\circ}$ and the elbows flexed to $90^{\circ}$. And the operative table was flexed to maximize the intercostal space. The first $1.5-\mathrm{cm}$ incision was selected in the eighth intercostal space in the midaxillary line, and was used for the camera (30 degree $10 \mathrm{~mm}$ high definition video thoracoscope). A 4-cm long incision was made in the third intercostal space in the preaxillary line (Figure 3). A third $1.5-\mathrm{cm}$ incision was performed in the ninth intercostal space in the post-axillary line for assistant.

First, pulmonary ligament and the entire right hilum was mobilized with combination of sharp and blunt dissection. Second, Pulmonary vein is the most forward hilar structure, sometimes immediately prior pulmonary trunk. The right upper lobe vein was transected with a vascular stapler (ETHICON $45 \mathrm{~mm}$ white stapler). Third, Truncus and posterior ascending pulmonary artery were then divided and transected with a vascular stapler. Injury of the adjacent structures like azygos vein, phrenic and recurrent laryngeal nerves must be avoided during this step. Fourth, major and minor fissures were stapled by $60 \mathrm{~mm}$ green linear stapler. Following clearance of the mediastinal lymph nodes of level 7 , the bronchial sleeve resection and reconstruction began. Excision of these lymph nodes will facilitate exposure of the bronchus. The distal right main bronchus and bronchus intermedius were fully mobilized to ensure adequate surgical exposure. Traction sutures were routinely placed on the lateral walls to reduce tension. Attention was then turned to bronchial anastomosis. Interrupted sutures were chosen for bronchial anastomosis. Bronchial membrane was sutured first, and then circumference end-to-end anastomoses were carried out using 3-0 absorbable sutures. The specimen was removed with a bag, and two sides of the bronchial resection margins were confirmed negative by frozen pathology. Afterwards, level 4R, 2R, and 3A were cleared (Figure 4). Bronchial anastomosis was confirmed without air leakage by water test. Finally, a 32-F chest tube was placed from the $1.5 \mathrm{~cm}$ incision (eighth intercostal space in the midaxillary line). Final pathology revealed T3N0M0 stage IIA squamous carcinoma.

Postoperative chest X-ray showed right middle and lower lobe reexpanded. Analgesia, antibiotics are used for three days. There were no complications and the patient was discharged 8 days postoperatively.

\section{Comments}

The incision of third intercostal space in the anterior axillary line can reduce the distance and angle between the 


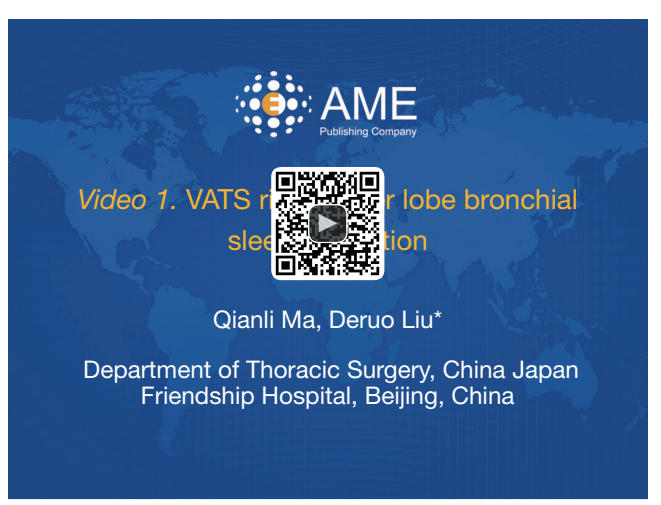

Figure 4 VATS right upper lobe bronchial sleeve resection (17). Available online: http://www.asvide.com/articles/1109

anastomosis and chest wall. Clearance of the mediastinal lymph nodes before cutting the bronchus was helpful for good exposure of the right mainstem bronchus, upper lobe bronchus and bronchus intermedius. And this approach would avoid pulling bronchial anastomosis during mediastinal lymph nodes clearance. Interrupted suture was safe and effective for VATS bronchial anastomosis.

\section{Acknowledgements}

None.

\section{Footnote}

Conflicts of Interest: The authors have no conflicts of interest to declare.

\section{References}

1. Jemal A, Bray F, Center MM, et al. Global cancer statistics. CA Cancer J Clin 2011;61:69-90.

2. Wain JC. Bronchoplastic Resections. In: Kaiser LR, editor. Mastery of Cardiothoracic Surgery. Philadelphia: Lippincott-Raven, 1998:68-76.

3. Ma Z, Dong A, Fan J, et al. Does sleeve lobectomy concomitant with or without pulmonary artery reconstruction (double sleeve) have favorable results for non-small cell lung cancer compared with pneumonectomy? A meta-analysis. Eur J Cardiothorac Surg 2007;32:20-8.

4. Nakanishi R, Yamashita T, Muranaka K, et al. Thoracoscopic carinal resection and reconstruction in a patient with mucoepidermoid carcinoma. J Thorac
Cardiovasc Surg 2013;145:1134-5.

5. Xu X, Chen H, Yin W, et al. Thoracoscopic half carina resection and bronchial sleeve resection for central lung cancer. Surg Innov 2014;21:481-6.

6. Jiao W, Zhu D, Cheng Z, et al. Thoracoscopic tracheal resection and reconstruction for adenoid cystic carcinoma. Ann Thorac Surg 2015;99:e15-7.

7. Zhao G, Dong C, Yang M, et al. Totally thoracoscopic tracheoplasty for a squamous cell carcinoma of the mediastinal trachea. Ann Thorac Surg 2014;98:1109-11.

8. Nakanishi K, Kuruma T. Video-assisted thoracic tracheoplasty for adenoid cystic carcinoma of the mediastinal trachea. Surgery 2005;137:250-2.

9. Zhang R, Xia W, Yu Z, et al. Complete video-assisted thoracic surgery right upper lobe sleeve resection. J Thorac Dis 2013;5 Suppl 3:S331-2.

10. Yang R, Shao F, Cao H, et al. Bronchial anastomosis using complete continuous suture in video-assisted thoracic surgery sleeve lobectomy. J Thorac Dis 2013;5 Suppl 3:S321-2.

11. Swanson SJ, Herndon JE 2nd, D'Amico TA, et al. Videoassisted thoracic surgery lobectomy: report of CALGB 39802--a prospective, multi-institution feasibility study. J Clin Oncol 2007;25:4993-7.

12. Kim K, Kim HK, Park JS, et al. Video-assisted thoracic surgery lobectomy: single institutional experience with 704 cases. Ann Thorac Surg 2010;89:S2118-22.

13. Nakanishi K. Video-assisted thoracic surgery lobectomy with bronchoplasty for lung cancer: initial experience and techniques. Ann Thorac Surg 2007;84:191-5.

14. Mahtabifard A, Fuller CB, McKenna RJ Jr. Video-assisted thoracic surgery sleeve lobectomy: a case series. Ann Thorac Surg 2008;85:S729-32.

15. Kamiyoshihara $M$, Nagashima T, Igai H, et al. Videoassisted thoracic lobectomy with bronchoplasty for lung cancer, with special reference to methodology. Interact Cardiovasc Thorac Surg 2011;12:534-8.

16. Schmid T, Augustin F, Kainz G, et al. Hybrid videoassisted thoracic surgery-robotic minimally invasive right upper lobe sleeve lobectomy. Ann Thorac Surg 2011;91:1961-5.

17. Ma Q, Liu D. VATS right upper lobe bronchial sleeve resection. Asvide 2016;3:340. Available online: http://www. asvide.com/articles/1109

Cite this article as: Ma Q, Liu D. VATS right upper lobe bronchial sleeve resection. J Thorac Dis 2016;8(8):2269-2271. doi: $10.21037 /$ jtd.2016.04.12 